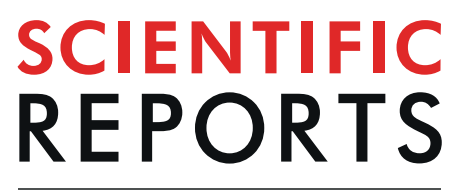

natureresearch

Check for updates

\title{
Targeted metabolomic analysis of serum amino acids in the adult Fontan patient with a dominant left ventricle
}

\author{
Miriam Michel $\mathbb{1}^{1,2}$, Karl-Otto Dubowy ${ }^{2}$, Andreas Entenmann ${ }^{3}$, Daniela Karall ${ }^{4}$, \\ Mark Gordian Adam ${ }^{5}$, Manuela Zlamy $\mathbb{1}^{4}$, Irena Odri Komazec ${ }^{1}$, Ralf Geiger ${ }^{1}$, \\ Christian Niederwanger ${ }^{4}$, Christina Salvador ${ }^{4}$, Udo Müller ${ }^{5}$, Kai Thorsten Laser $^{2,6}$ \& \\ Sabine Scholl-Bürgi ${ }^{4,6}$
}

Growing interest lies in the assessment of the metabolic status of patients with a univentricular circulation after Fontan operation, especially in changes of amino acid metabolism. Using targeted metabolomic examinations, we investigated amino acid metabolism in a homogeneous adult Fontanpatient group with a dominant left ventricle, seeking biomarker patterns that might permit better understanding of Fontan pathophysiology and early detection of subtle ventricular or circulatory dysfunction. We compared serum amino acid levels ( 42 analytes; AbsolutelDO p180 kit, Biocrates Life Sciences, Innsbruck, Austria) in 20 adult Fontan patients with a dominant left ventricle and those in age- and sex-matched biventricular controls. Serum concentrations of asymmetric dimethylarginine, methionine sulfoxide, glutamic acid, and trans-4-hydroxyproline and the methionine sulfoxide/ methionine ratio (Met-SO/Met) were significantly higher and serum concentrations of asparagine, histidine, taurine, and threonine were significantly lower in patients than in controls. Met-SO/ Met values exhibited a significant negative correlation with oxygen uptake during exercise. The alterations in amino acid metabolome that we found in Fontan patients suggest links between Fontan pathophysiology, altered cell energy metabolism, oxidative stress, and endothelial dysfunction like those found in biventricular patients with congestive heart failure. Studies of extended amino acid metabolism may allow better understanding of Fontan pathophysiology that will permit early detection of subtle ventricular or circulatory dysfunction in Fontan patients.

Ventricular dysfunction and circulatory failure with progressing end-organ impairment like renal or liver dysfunction are an important cause of morbidity and mortality in adults with complex congenital heart disease (CHD), especially in patients with single-ventricle types of CHD and Fontan circulation ${ }^{1,2}$. Besides limited cardiac output, alterations that mark Fontan hemodynamics are passive flow to the lungs, chronically elevated venous pressures, and congestion. Unfortunately, the clinical use of traditional markers such as N-terminal prohormone of brain natriuretic peptide (NT-proBNP) levels for non-invasive diagnostics and monitoring in such patients is limited ${ }^{3,4}$. Thus, for early detection of cardiac and circulatory derangement and for evaluation and tailoring of treatment options, regular functional assessment of these patients is crucial, with complete clinical examination, electrocardiogram, imaging studies, determination of values for traditional laboratory markers, or exercise capacity testing. In adult biventricular patients, novel candidate biomarkers, in addition to natriuretic

\footnotetext{
${ }^{1}$ Department of Pediatrics III, Division of Pediatric Cardiology, Medical University of Innsbruck, Anichstraße 35, 6020, Innsbruck, Austria. ${ }^{2}$ Center of Pediatric Cardiology and Congenital Heart Disease, Heart and Diabetes Center North Rhine-Westphalia, Ruhr-University of Bochum, Georgstraße 11, 32545, Bad Oeynhausen, Germany. ${ }^{3}$ Department of Pediatrics I, Division of Gastroenterology and Hepatology, Medical University of Innsbruck, Anichstraße 35, 6020, Innsbruck, Austria. ${ }^{4}$ Department of Pediatrics I, Division of Inherited Metabolic Disorders, Medical University of Innsbruck, Anichstraße 35, 6020, Innsbruck, Austria. ${ }^{5}$ Biocrates Life Sciences AG, Eduard-Bodem-Gasse 8, 6020, Innsbruck, Austria. ${ }^{6}$ These authors jointly supervised this work: Kai Thorsten Laser and Sabine Scholl-Bürgi. ${ }^{\bowtie}$ e-mail: miriam.michel@i-med.ac.at
} 


\begin{tabular}{|c|c|c|c|}
\hline & Fontan patients & Controls & P-value \\
\hline Total [n] & 20 & 20 & \\
\hline Female sex [n] & 7 & 7 & \\
\hline Age [years] & $23.1 \pm 5.1$ & $24.7 \pm 6.6$ & 0.28 \\
\hline After TCPC [years] & $18.8 \pm 5.2$ & & \\
\hline Bodyweight $[\mathrm{kg}]$ & $69.8 \pm 13.2$ & $73.3 \pm 11.7$ & 0.17 \\
\hline Height $[\mathrm{cm}]$ & $171.3 \pm 7.4$ & $174.5 \pm 8.7$ & 0.04 \\
\hline Body mass index $\left[\mathrm{kg} / \mathrm{m}^{2}\right]$ & $23.8 \pm 4.1$ & $22.5 \pm 3.3$ & 0.05 \\
\hline $\mathrm{SpO}_{2}$ at rest [\%] & $93 \pm 3$ & $99 \pm 1$ & $<0.00001$ \\
\hline $\mathrm{SpO}_{2}$ at exercise [\%] & $90 \pm 3$ & $98 \pm 1$ & $<0.00001$ \\
\hline$\dot{\mathrm{V}} \mathrm{O}_{2}$ at rest $[\mathrm{mL} / \mathrm{kg} / \mathrm{min}]$ & $5.6 \pm 1.7$ & $5.8 \pm 1.1$ & 0.03 \\
\hline$\dot{\mathrm{V}} \mathrm{O}_{2} \mathrm{AT}[\mathrm{mL} / \mathrm{kg} / \mathrm{min}]$ & $24.5 \pm 4.9$ & $30.1 \pm 3.6$ & $<0.00001$ \\
\hline Peak $\dot{V}_{2}[\mathrm{~mL} / \mathrm{kg} / \mathrm{min}]$ & $28.8 \pm 10.1$ & $45.7 \pm 6.4$ & $<0.00001$ \\
\hline Double inlet left ventricle [n] & 10 & & \\
\hline $\mathrm{TA}+\mathrm{PS} / \mathrm{PA}[\mathrm{n}]$ & 9 & & \\
\hline $\mathrm{TA}+\mathrm{PS}+\mathrm{VSD}[\mathrm{n}]$ & 1 & & \\
\hline Extracardiac Fontan [n] & 16 & & \\
\hline Open fenestration (at study) [n] & 3 & & \\
\hline LPA dilation/stent [n] & 4 & & \\
\hline Tunnel dilation/stent [n] & 6 & & \\
\hline Closure of fenestration [n] & 1 & & \\
\hline Closure of vv collateral [n] & 3 & & \\
\hline Electrophysiologic examination [n] & 2 & & \\
\hline
\end{tabular}

Table 1. Participants' clinical characteristics ${ }^{9}$. Values are given as mean \pm standard deviation. AT, anaerobic threshold; LPA, left pulmonary artery; n, number; PA, pulmonary atresia; PS, pulmonary stenosis; $\mathrm{SpO}_{2}$, pulsoxymetric oxygen saturation; TA, tricuspid atresia; TCPC, total cavopulmonary connection; $\dot{\mathrm{VO}}_{2}$, oxygen uptake; VSD, ventricular septal defect; vv, veno-venous.

peptides and troponins, for congestive heart failure and vascular perturbations have been identified via metabolomics, the study of small organic molecules, their synthesis, and their breakdown ${ }^{5,6}$. Interest has grown recently in the metabolic status of Fontan patients: besides reported abnormalities in Fontan patients' glucose metabolism $^{7}$, to date their handling of lipids has been best studied, with important changes shown, especially in the cholesterol, lipoprotein, and phospholipid pathways, hinting at chronic low-level inflammation and at altered cell signalling and energy metabolism as are found in biventricular patients with congestive heart failure ${ }^{8,9}$. In the biventricular patient with heart failure, alterations occur especially in the handling of amino acids important in both myocardium protein turnover and energy metabolism ${ }^{10}$. Additionally, the Fontan-specific characteristic of chronically elevated venous pressure favors development of a protein losing enteropathy together with end-organ, especially liver, dysfunction ${ }^{2}$. Thus, study of the metabolism of amino acids also should be a promising field in Fontan patients. With the help of targeted metabolomic examinations we hence elected to investigate amino acid metabolism in a homogeneous adult Fontan-patient group with a dominant left ventricle, seeking biomarker patterns that might permit a better understanding of Fontan pathophysiology or early detection of ventricular or circulatory dysfunction.

\section{Results}

After applying all inclusion and exclusion criteria, 20 adult Fontan patients with a systemic left ventricle were selected for the study (Supplemental Fig. 1). The results of "traditional" examinations (patient and control clinical assessment, exercise capacity testing, routine laboratory analyses) are set out in our recent work on lipid metabolism $^{9}$. Table 1 lists clinical and exercise capacity testing parameters, showing as major features that in Fontan patients, minimum (at exercise) and maximum (at rest) pulse-oximeter oxygen saturation as well as $\dot{\mathrm{V}} \mathrm{O}_{2}$ at the anaerobic threshold and at maximum were significantly lower than in controls.

Routine analytes. Hematocrit, hemoglobin concentrations ("hemoglobin"), gamma glutamyl transferase and alanine aminotransferase activities, total bilirubin and creatinine concentrations ("total bilirubin" and "creatinine"), and international normalized ratio (INR) values and triglyceride and high density lipoprotein-cholesterol (HDL-C) concentrations ("triglycerides" and "HDL-C") differed significantly between Fontan patients and controls (Table 2).

Metabolomic examination of serum amino acids. Serum concentrations of 29 amino acids or biogenic amines and their derivatives were determined, with selected sums and ratios (Table 3, Fig. 1). In Fontan patients serum concentrations of methionine (Met) sulfoxide (Met-SO), asymmetric dimethyl arginine (ADMA), glutamic acid, and trans-4-hydroxyproline and the ratio of Met-SO to Met (Met-SO/Met) were significantly higher, and serum concentrations of asparagine, histidine, taurine, and threonine were significantly lower than in controls. 


\begin{tabular}{|l|l|l|l|}
\hline & Fontan patients & Controls & P-value \\
\hline Hematocrit [\%] & $47.8 \pm 5.6$ & $39.3 \pm 4.2$ & $<0.00001 \uparrow$ \\
\hline Hemoglobin [g/dL] & $16.4 \pm 2.1$ & $12.7 \pm 1.4$ & $<0.00001 \uparrow$ \\
\hline Total cholesterol [mg/dL] & $145.3 \pm 26.5$ & $149 \pm 34.2$ & 0.77 \\
\hline HDL-C [mg/dL] & $42.5 \pm 15.9$ & $51.3 \pm 12.3$ & $0.03 \downarrow$ \\
\hline Non-HDL-C [mg/dL] & $85.2 \pm 24.8$ & $73.1 \pm 20.8$ & 0.2 \\
\hline Triglycerides [mg/dL] & $128.6 \pm 86.5$ & $47.3 \pm 22.8$ & $0.003 \uparrow$ \\
\hline Total protein [g/dL] & $7.2 \pm 0.5$ & $7.0 \pm 0.7$ & 0.31 \\
\hline Albumin [mg/dL] & $4145 \pm 492$ & $4215 \pm 208$ & 0.64 \\
\hline Creatinine [mg/dL] & $0.8 \pm 0.12$ & $0.53 \pm 0.18$ & $<0.00001 \uparrow$ \\
\hline Total bilirubin [mg/dL] & $1.22 \pm 0.67$ & $0.3 \pm 0.29$ & $<0.00001 \uparrow$ \\
\hline AST [U/L] & $35.3 \pm 7.7$ & $31.6 \pm 8.4$ & 0.12 \\
\hline ALT [U/L] & $39.4 \pm 11.4$ & $31.9 \pm 10.1$ & $0.04 \uparrow$ \\
\hline gGT [U/L] & $86.5 \pm 43.6$ & $35.1 \pm 19.4$ & $0.00002 \uparrow$ \\
\hline INR & $2.1 \pm 0.76$ & $1.02 \pm 0.04$ & $<0.00001 \uparrow$ \\
\hline NT-proBNP [pg/mL] & $52.4 \pm 69.2$ & $39.3 \pm 30.4$ & 0.88 \\
\hline CRP [mg/dL] & $0.18 \pm 0.2$ & $0.16 \pm 0.14$ & 0.47 \\
\hline
\end{tabular}

Table 2. Values of routine analytes and of amino acids or biogenic amines and their derivatives. Values are given as mean \pm standard deviation. ALT, alanine aminotransferase; AST, aspartate aminotransferase; CRP, C-reactive protein; dL, decilitre; g, gram; gGT, gamma glutamyl transferase; HDL-C, high density lipoproteincholesterol; INR, international normalized ratio; NT-proBNP, N-terminal prohormone of brain natriuretic peptide; U, unit; $\uparrow$, statistically significant higher serum concentration in Fontan patients than in controls; $\downarrow$, statistically significant lower serum concentration in Fontan patients than in controls.

Correlation of routine biochemical and clinical findings with metabolomic parameters. Among routine analytes, the variables hemoglobin, albumin, and triglycerides displayed significantly positive correlations with glutamic acid. Creatinine and triglycerides displayed significantly positive correlations with ADMA, as did the variables creatinine and total bilirubin with Met-SO. Hematocrit, hemoglobin, and INR as well as minimum and maximum oxygen saturations revealed significantly positive correlations with Met-SO/Met values, and oxygen uptake at the anaerobic threshold and maximum oxygen uptake displayed significantly negative correlations with Met-SO/Met values. Single significant correlations between isolated variables included positive correlation of albumin with ornithine and serine, of triglycerides with alpha aminoadipic acid, and of the oxygen uptake at the anaerobic threshold with threonine as well as negative correlations of alanine aminotransferase activity with taurine and of INR with serotonin. No further correlations were identified, especially none with CRP or NT-proBNP (Supplemental Table 1).

\section{Discussion}

To the best of our knowledge, our study is the first clinical metabolomics study focusing on Fontan patients' serum amino acid patterns. Its main finding is that, in comparison with controls, adult Fontan patients with a morphologically left dominant ventricle exhibit a distorted amino acid metabolome, hinting at altered (myocardial) cell energy metabolism and an elevated myocardial protein turnover as well as at oxidative stress and endothelial dysfunction, as found in biventricular patients with congestive heart failure.

Altered cell energy metabolism and elevated myocardial protein turnover. Decreased serum concentrations of taurine, asparagine, and threonine and increased concentrations of glutamic acid and hydroxyproline in our Fontan patients indicate alterations in (myocardial) cell energy metabolism and elevated myocardium protein turnover, as found in biventricular patients with congestive heart failure. Taurine is abundant in myocardial tissue, with a major function in regulation of the respiratory chain: the taurine-deficient heart suffers impaired respiratory chain function and diminished long chain fatty acid uptake by mitochondria, as found in patients with congestive heart failure ${ }^{10}$. Decreased concentrations of taurine are described in patients with dilated cardiomyopathy and in golden retrievers with heart failure ${ }^{10,11}$, and many studies report a beneficial effect of taurine supplementation on myocardial function in patients with congestive heart failure ${ }^{12}$.

Glutamic acid and asparagine also take part in central pathways of aerobic cell respiration and thereby of energy production. Via anaplerotic reactions, both amino acids are substantially involved in the tricarboxylic acid cycle $^{13}$. Against the background of a switch in heart failure of preferred myocardial energy substrate from fatty acids to glucose and ketone bodies ${ }^{14}$, our findings hint at altered myocardial energy metabolism, indicating subtle ventricular dysfunction. This idea is supported by glutamic acid's involvement in the synthesis of metabolites, e.g., by serving as a precursor for the biosynthesis of amino acids such as proline and arginine ${ }^{15,16}$. Indeed, increased serum levels of glutamic acid indicate increased protein turnover, as typical in adults with congestive heart failure or coronary heart disease $\mathrm{e}^{17,18}$. The same is true for threonine, a major component of proteins, e.g., of collagen and immunoglobulins: We found decreased serum threonine levels, and decreased plasma levels are described in patients with chronic heart failure ${ }^{17}$. The increase that we observed in hydroxyproline, an analyte used to track collagen degradation, also reflects elevated protein turnover: Elevated urine and serum hydroxyproline levels are reported after muscle damage or in the bedridden and elderly ${ }^{19,20}$. 


\begin{tabular}{|c|c|c|c|c|c|c|}
\hline Metabolite & HMBD ID & Patients & Controls & p-value & q-value & mean FC \\
\hline Ac-Orn & HMDB0003357 & NA & NA & NA & NA & NA \\
\hline ADMA & HMDB01539 & $0.51 \pm 0.12$ & $0.41 \pm 0.05$ & 0.0004 & $0.002 \uparrow$ & 1.26 \\
\hline Alanine & HMDB00161 HMDB01310 & $401.4 \pm 84.2$ & $410.5 \pm 123.0$ & 0.99 & 0.99 & -1.02 \\
\hline alpha-AAA & HMDB00510 & $1.12 \pm 0.61$ & $0.77 \pm 0.44$ & 0.03 & 0.06 & 1.44 \\
\hline Arginine & HMDB00517 HMDB03416 & $91.7 \pm 26.8$ & $108.2 \pm 21.8$ & 0.03 & 0.06 & -1.18 \\
\hline Asparagine & HMDB00168 HMDB003378 & $43.7 \pm 7.7$ & $50.7 \pm 8.0$ & 0.007 & $0.016 \downarrow$ & -1.16 \\
\hline Aspartic acid & HMDB00191 HMDB06483 & $18.0 \pm 10.4$ & $17.2 \pm 6.9$ & 0.93 & 0.96 & 1.05 \\
\hline c4-OH-Pro & HMDB0240251 & NA & NA & NA & NA & NA \\
\hline Carnosine & HMDB00033 & NA & NA & NA & NA & NA \\
\hline Citrulline & HMDB00904 & $29.4 \pm 6.3$ & $29.2 \pm 6.6$ & 0.91 & 0.95 & 1.01 \\
\hline DOPA & HMDB00181 HMDB00609 & NA & NA & NA & NA & NA \\
\hline Dopamine & HMDB00073 & NA & NA & NA & NA & NA \\
\hline Glutamine & HMDB00641 HMDB03423 & $717.4 \pm 134.1$ & $749.0 \pm 111.7$ & 0.38 & 0.47 & -1.04 \\
\hline Glutamic acid & HMDB00148 HMDB03339 & $85.9 \pm 63.4$ & $47.0 \pm 21.8$ & 0.0006 & $0.002 \uparrow$ & 1.83 \\
\hline Glycine & HMDB00123 & $321.6 \pm 74.1$ & $345.3 \pm 91.7$ & 0.41 & 0.5 & -1.07 \\
\hline Histidine & HMDB00177 & $93.1 \pm 15$ & $110.4 \pm 24.4$ & 0.008 & $0.02 \downarrow$ & -1.19 \\
\hline Histamine & HMDB00870 & NA & NA & NA & NA & NA \\
\hline Isoleucine & HMDB00172 HMDB0000557 & $106.6 \pm 31.6$ & $100.9 \pm 39.5$ & 0.44 & 0.53 & 1.06 \\
\hline Kynurenine & HMDB00684 & NA & NA & NA & NA & NA \\
\hline Leucine & HMDB00687 HMDB0013773 & $222.9 \pm 78.6$ & $232.8 \pm 106.6$ & 0.99 & 0.99 & -1.04 \\
\hline Lysine & HMDB00182 HMDB03405 & $168.1 \pm 23.5$ & $165.8 \pm 32.4$ & 0.69 & 0.79 & 1.01 \\
\hline Methionine & HMDB00696 & $27.9 \pm 8.3$ & $29.4 \pm 7.8$ & 0.47 & 0.55 & -1.06 \\
\hline Met-SO & HMDB02005 & $0.94 \pm 0.32$ & $0.58 \pm 0.23$ & 0.00008 & $0.0005 \uparrow$ & 1.64 \\
\hline Nitro-Tyr & HMDB01904 & NA & NA & NA & NA & NA \\
\hline Ornithine & HMDB00214 HMDB03374 & $139.4 \pm 82.3$ & $111.6 \pm 40.9$ & 0.22 & 0.29 & 1.25 \\
\hline PEA & HMDB0012275 & NA & NA & NA & NA & NA \\
\hline Phenylalanine & HMDB00159 & $77.7 \pm 16.9$ & $77.0 \pm 12.9$ & 0.99 & 0.99 & 1.01 \\
\hline Proline & HMDB00162 HMDB03411 & $259.4 \pm 63.7$ & $265.7 \pm 61.6$ & 0.73 & 0.81 & -1.02 \\
\hline Putrescine & HMDB01414 & NA & NA & NA & NA & NA \\
\hline Sarcosine & HMDB00271 & $1.57 \pm 0.94$ & $1.62 \pm 0.79$ & 0.73 & 0.81 & -1.03 \\
\hline SDMA & HMDB03334 & $0.46 \pm 0.06$ & $0.42 \pm 0.08$ & 0.1 & 0.15 & 1.08 \\
\hline Serine & HMDB00187 HMDB03406 & $158.4 \pm 33.3$ & $146.5 \pm 23.7$ & 0.21 & 0.29 & 1.08 \\
\hline Serotonin & HMDB00259 & $0.56 \pm 0.33$ & $0.7 \pm 0.21$ & 0.03 & 0.06 & -1.26 \\
\hline Spermidine & HMDB01257 & NA & NA & NA & NA & NA \\
\hline Spermine & HMDB01256 & NA & NA & NA & NA & NA \\
\hline t4-OH-Pro & HMDB00725 HMDB0006055 & $15.8 \pm 5.06$ & $11.7 \pm 5.2$ & 0.007 & $0.02 \uparrow$ & 1.35 \\
\hline Taurine & HMDB00251 & $100.4 \pm 57.1$ & $132.6 \pm 56.54$ & 0.02 & $0.049 \downarrow$ & -1.32 \\
\hline Threonine & HMDB04041 HMDB00167 & $105.6 \pm 21.0$ & $127.3 \pm 28.4$ & 0.008 & $0.02 \downarrow$ & -1.21 \\
\hline Tryptophan & HMDB00929 HMDB0013609 & $90.5 \pm 20.2$ & $89.5 \pm 23.3$ & 0.84 & 0.89 & 1.01 \\
\hline Tyrosine & HMDB00158 & $87.0 \pm 23.0$ & $83.6 \pm 19.4$ & 0.69 & 0.79 & 1.04 \\
\hline Valine & HMDB00883 & $298.9 \pm 72.5$ & $290.7 \pm 76.9$ & 0.7 & 0.79 & 1.03 \\
\hline BCAA & & $628 \pm 174$ & $624 \pm 217$ & 0.8 & 0.87 & 1.01 \\
\hline AAA & & $255 \pm 53$ & $250 \pm 47$ & 0.8 & 0.87 & 1.02 \\
\hline Total AA & & $3544 \pm 466$ & $3588 \pm 682$ & 0.93 & 0.96 & -1.01 \\
\hline Essential AA & & $1098 \pm 223$ & $1113 \pm 293$ & 0.97 & 0.99 & -1.01 \\
\hline Fischer ratio & & $2.47 \pm 0.52$ & $2.45 \pm 0.5$ & 0.9 & 0.95 & 1.01 \\
\hline Met-SO/Met & & $0.04 \pm 0.016$ & $0.02 \pm 0.007$ & $<0.00001$ & $0.0001 \uparrow$ & 1.78 \\
\hline
\end{tabular}

Table 3. Values of amino acids or biogenic amines and their derivatives, and of selected sums and ratios. Metabolite concentrations for all analytes with selected sums and ratios, grouped by Fontan patients vs. controls. Values are given as mean \pm standard deviation, unit of values of data $[\mu \mathrm{mol} / \mathrm{l}]$. FC, fold change (Fontan patients vs. controls); HMBD ID, Human Metabolome Database identification; AA, amino acids; AAA, aromatic amino acids; Ac-Orn, acetylornithine; ADMA, asymmetric dimethylarginine; alpha-AAA, alpha aminoadipic acid; BCAA, branched-chain amino acids; c4-OH-Pro, cis-4-hydroxyproline; DOPA, dihydroxyphenylalanine; Met-SO, methionine sulfoxide; Nitro-Tyr, nitrotyrosine; PEA, phenylethylamine; SDMA, symmetric dimethylarginine; t4-OH-Pro, trans-4-hydroxyproline; $\uparrow$, statistically significant higher serum concentration in Fontan patients than in controls; $\downarrow$, statistically significant lower serum concentration in Fontan patients than in controls. 'Fischer ratio' is the ratio of BCAA/AAA. 

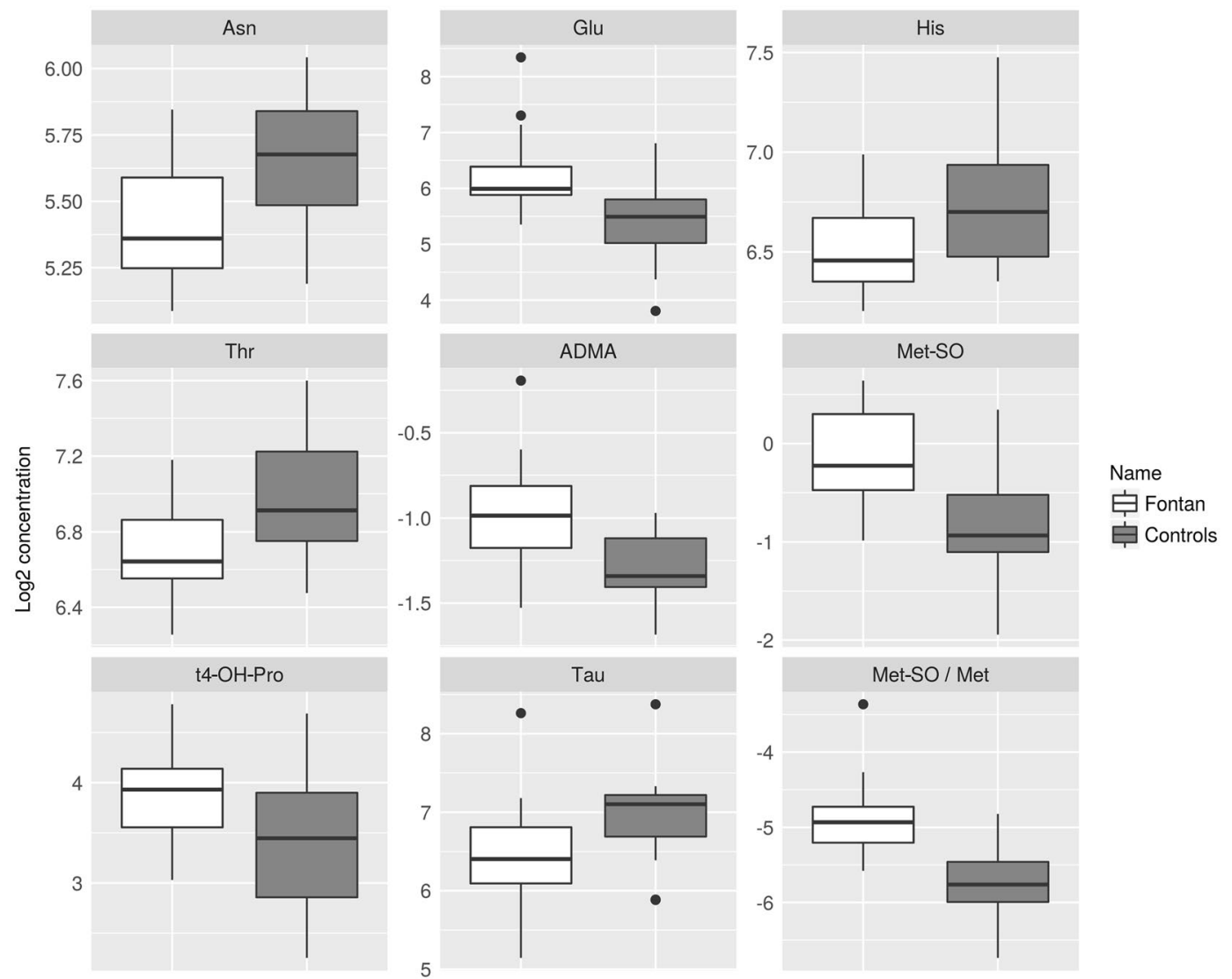

Figure 1. Box-and-whisker plots of serum concentrations of amino acids or biogenic amines and their ratios that differed significantly between Fontan patients (grey boxes) and controls (white boxes). The boxes show the $1^{\text {st }}(\mathrm{Q} 1)$ and $3^{\text {rd }}$ quartile (Q3), the whiskers the minimum and the maximum. Black circles represent outlying values as identified by the interquartile range (IQR) rule (values smaller than (Q1-1.5*IQR) or values larger than $(\mathrm{Q} 3+1.5 * \mathrm{IQR})$ ). ADMA, asymmetric dimethylarginine; Asn, asparagine; Glu, glutamic acid; His, histidine; Met, methionine; Met-SO, methionine sulfoxide; Met-SO/Met, methionine sulfoxide/methionine ratio; t4-OH-Pro, trans-4-hydroxyproline; Tau, taurine; Thr, threonine.

Oxidative stress and endothelial dysfunction. Protein turnover also is elevated in the presence of hyperreactive oxygen species, i.e., under oxidative stress and during endothelial dysfunction, both of which are directly linked to heart failure: Nitrous oxide (NO) synthase (NOS) isoforms are expressed not only in endothelial cells but also in cardiomyocytes, and NO regulates cardiac function through vascular-dependent and -independent effects, with, in the healthy heart, a positive inotropic effect at low NO exposure and a negative one at higher exposure ${ }^{21,22}$. In heart failure, regulation of myocardial NO production and release breaks down, with excessive release, and peripheral and vascular endothelial NOS activity is lost, resulting in endothelial dysfunction with decreased NO bioavailability attributable to increased oxidative stress ${ }^{23}$. In the course of cardiac decompensation, NO likely influences several of the core features of cardiac failure, e.g., chamber dilation, defective b-adrenergic responsiveness, and calcium cycling ${ }^{22}$.

That serum concentrations of ADMA were elevated also suggests that our Fontan patients were under oxidative stress, with altered endothelial function, consistent with subtle ventricular dysfunction. ADMA, a methyl derivate of arginine, is involved in NO-signalling and in pro- and antioxidant and -inflammatory processes. It is the major endogenous inhibitor of nitric oxide synthase (NOS). By displacing arginine, the normal NOS substrate, ADMA enhances oxidative stress by influencing NO-reactive oxygen species balance and disturbs vasodilation $^{21,24-26}$. ADMA values reportedly track enhanced cardiovascular risk with endothelial dysfunction ${ }^{27,28}$, positively correlating with age, mean arterial pressure, renovascular resistance, intimal media thickness, and peripheral arterial occlusive disease $\mathrm{e}^{5,29-33}$, ADMA is additionally involved in further nitric oxide (NO)-dependent signalling processes, interfering with anti-thrombotic, anti-inflammatory, and anti-apoptotic actions ${ }^{21}$.

The increases in value for Met-SO and for the Met-SO/Met ratio as indicators of systemic oxidative stress also suggest that our patients are under oxidative stress and suffer from endothelial dysfunction. Reaction with oxygen species yields Met-SO, which activates endogenous antioxidant enzymes such as Met-SO reductase A and induces synthesis of glutathione, thereby counteracting oxidative stress and inflammation ${ }^{34}$. Increased Met-SO levels have been reported in vascular disease, cardiac ischemia, and in left ventricular diastolic dysfunction ${ }^{35,36}$. The negative correlation observed between Met-SO/Met values and oxygen uptake under exercise strikingly emphasizes the role of markers of oxidative stress as indicators for subtle ventricular dysfunction in our patients. Finally, our hypothesis that oxidative stress affects our patients is supported by the decreased level found of histidine, with 
its anti-oxidant and anti-inflammatory properties ${ }^{37}$, and by decreases in HDL-C ${ }^{9,38-40}$. A direct interplay of lipoprotein metabolism and markers for oxidative stress exists: lipoprotein disorders are associated with increased ADMA $^{41}$

ADMA also has been used to track heart failure in patients with $\mathrm{CHD}$, and with regard to heart failure is even more sensitive than NT-proBNP. However, only $13 \%$ of the patients studied had univentricular heart disease; in addition, patient exercise capacity was lower than that in our patient group ${ }^{33}$. Thus we stress that our findings of altered amino acid serum levels might imply subtle rather than frank heart failure ${ }^{42,43}$, a hypothesis supported by the fact that we found no correlation of any of the metabolites with the traditional marker for heart failure, NT-proBNP, nor with the traditional marker for inflammation C-reactive protein, and by the fact that neither by traditional clinical-laboratory means nor by metabolomic (Fischer ratio) criteria did we find any evidence for important (Fontan-associated) liver disease in our patients. Given that many adult Fontan patients will develop ventricular dysfunction, to hypothesise subtle heart failure in our patient group might well be appropriate ${ }^{1}$. Still, with respect to the definition of heart failure, which is the "inability of the heart to meet resting and exercise demands at low filling pressures"2, we cannot exclude with certainty that the metabolic alterations delineated solely reflect Fontan-specific pathophysiology with its circulatory abnormalities, with paramount abnormal systemic or pulmonary endothelial function, and with a tendency towards the formation of veno-venous or aortopulmonary collaterals ${ }^{44-48}$.

To examine the amino acid profile of Fontan patients with frank ventricular, circulatory, or hepatic impairment and to correlate increases in serum ADMA and Met-SO content with direct measurement of endothelin or with collateral vessel flow via magnetic resonance imaging studies thus would be of interest ${ }^{49,50}$.

Limitations. Because Fontan patients are few, the studied group size is small, possibly limiting the extent to which our findings can be generalized. Also limiting may be our choice of a targeted metabolomic approach and a commercial kit, as metabolites not assessed may be important. That we analyzed serum samples precludes direct comparison between our results and those of studies that used tissue samples, and we acknowledge that having assayed serum rather than vascular or myocardial tissue, we cannot rule out certain system-driven aspects of disease pathogenesis: That is, unknown confounders that affect metabolic profiles might be the true basis for the observed differences. Moreover, differences in body composition or lifestyle parameters might have influenced our results to an unknown degree. We strove to lessen the likelihood of such errors by following a strict inclusion and exclusion protocol, especially with regard to (known) comorbidities or medication.

\section{Conclusion}

The striking alterations in amino acid profile that we found may link Fontan pathophysiology with altered cell energy metabolism, oxidative stress, and endothelial dysfunction as found in biventricular patients with congestive heart failure. Markers identified through mass spectrometry-based extended amino acid metabolism might thus complement traditional diagnostic tools such as imaging, exercise capacity testing, and traditional laboratory biomarker determinations, yielding a better understanding of Fontan pathophysiology, and they are promising candidates for the early detection of ventricular or circulatory dysfunction in Fontan patients.

\section{Methods}

Like our recently published results on phospholipid and acylcarnitine metabolomic examinations, this work is a subwork of the main study protocol (Trial registration number: ClinicalTrials.gov Identifier NCT03886935).

Patients. Between September 2016 and March 2017, we prospectively examined adult Fontan patients with a dominant left single ventricle and age- and sex-matched healthy biventricular controls at the Center of Pediatric Cardiology and Congenital Heart Disease, Heart and Diabetes Center North Rhine-Westphalia, Ruhr-University of Bochum, Germany ${ }^{9}$. All patients had undergone two-stage palliation with partial and total cavopulmonary anastomosis. None had had aortic reconstruction or aortopulmonary shunting. Table 4 shows inclusion and exclusion criteria. See the flow chart according to STROBE (Strengthening the Reporting of Observational Studies in Epidemiology [https://strobe-statement.org/index.php?id=strobe-home]) (Supplemental Fig. 1) for details on the flow of patients through the present study.

Clinical and laboratory examinations were performed as described in detail elsewhere ${ }^{9}$. Age, sex, weight, medication, and cardiac risk factor assessment, with blood sampling for routine hematological and biochemical profiling, was conducted during an outpatient visit. Fasting patients underwent phlebotomy while recumbent. Echocardiography followed, and, after a defined snack rich in carbohydrates, exercise capacity was tested. We correlated metabolic results with routine laboratory parameters and exercise capacity parameters ${ }^{51}$. All patients underwent symptom-limited treadmill exercise capacity testing with expiratory gas analysis ${ }^{52}$. A 12-lead ECG was used to determine heart rate. Oxygen uptake at rest $\left(\dot{\mathrm{VO}}_{2}\right.$ at rest, $\left.\mathrm{ml} / \mathrm{kg} / \mathrm{min}\right)$, at the anaerobic threshold $\left(\dot{\mathrm{VO}}_{2} \mathrm{AT}\right.$, $\mathrm{ml} / \mathrm{kg} / \mathrm{min}$ ), and maximum uptake of oxygen $\left(\dot{\mathrm{VO}}_{2} \max , \mathrm{ml} / \mathrm{kg} / \mathrm{min}\right)$ were measured.

Blood studies required samples $0.5 \mathrm{ml}$ greater than those for routine assessments to permit determinations of concentrations of amino acid-metabolism analytes. The blood sample was directly drawn into a tube containing a clotting activator and centrifuged within $20 \mathrm{~min}\left(15^{\circ} \mathrm{C}, 10 \mathrm{~min}, 2500 \times \mathrm{g}\right)$ for separation of serum. Serum aliquots were immediately frozen and stored at $-80^{\circ} \mathrm{C}$ for further analyses. Frozen samples were transported on dry ice to the analysing laboratory. Analyses were performed in batches of 10 samples $^{9}$.

Sample preparation. Before analysis, all serum samples were processed as described, with samples thawed on ice, then centrifuged; the supernatant was subjected to further analyses ${ }^{6,9}$. The AbsoluteIDQ p180 kit assay (Biocrates Life Sciences AG, Innsbruck, Austria) permitted targeted, fully automated quantification of 188 


\begin{tabular}{|l|l|}
\hline Inclusion criteria & Exclusion criteria \\
\hline Written informed consent & Medication affecting metabolic state \\
\hline Age $\geq 18$ years & Medication affecting hemodynamic state \\
\hline 8 hours fasting before blood sampling & Coronary artery disease \\
\hline Dominant left ventricle (patients) & Failure of systemic ventricle \\
\hline Biventricular healthy heart (controls) & Valvular stenosis \\
\hline & Atrioventricular regurgitation $>$ mild \\
\hline & Aortic regurgitation $>$ mild \\
\hline & Recurrent effusions \\
\hline & Protein-losing enteropathy \\
\hline & Metabolic disease \\
\hline & Malignancy \\
\hline & Other cachectic disease, malnutrition \\
\hline & Inflammatory disease \\
\hline & Myeloproliferative disorder \\
\hline & Pregnancy or lactation \\
\hline & Multiple organ failure \\
\hline & Mental or physical handicap precluding exercise \\
\hline & $\dot{\text { VO }} 2$ AT $<20 \mathrm{ml} / \mathrm{kg} /$ min \\
\hline
\end{tabular}

Table 4. Inclusion and exclusion criteria ${ }^{9}$. $\mathrm{V}_{2} \mathrm{AT}$, oxygen uptake at the anaerobic threshold.

analytes (42 amino acids or biogenic amines and their derivatives) based on phenylisothiocyanate derivatization in the presence of internal standards followed by liquid chromatography mass spectrometry using a TSQ-Vantage (Thermo Fisher Scientific, Waltham, MA) instrument with electrospray ionization.

Statistical analysis. To exclude metabolites below the limit of detection (LOD), the raw data $(\mu \mathrm{mol} / \mathrm{L})$ were cleaned applying a modified $80 \%$ rule; for statistical analysis at least $80 \%$ valid values above LOD needed to be available per analyte in the samples for each group. This reduced the dataset to 143 analytes ( 29 amino acids or biogenic amines and their derivatives). Remaining values below LOD were imputed applying a logspline method with values between LOD and LOD/2. After $\log 2$ transformation of metabolomics data as well as of routine analytes and clinical data the dataset underwent multivariate (hierarchical cluster analysis) and univariate statistical analyses. Student's t-testing with a Benjamini-Hochberg correction identified significant metabolite (and clinical routine-parameter) differences between patients and controls. P-values were calculated to identify significant changes between controls and Fontan patients, and were adjusted for multiple testing, or false discovery rate (FDR), according to Benjamini and Hochberg. To interpret the correlation, the significance of the correlation was calculated from $\mathrm{r}$ and from the degrees of freedom (a variable dependent on the sample number) (Supplemental Table 1). As a measure of linear correlation, $r$ can have values between -1 and 1 , where 1 indicates total positive linear correlation, 0 indicates no linear correlation, and -1 indicates total negative linear correlation. Correlations with FDR-adjusted p-values $<0.05$ and $r>0.5(<-0.5)$ were considered statistically significant; in Supplemental Table 1, table cells displaying a statistically significant correlation are highlighted by a green background ${ }^{9}$.

Ethical approval and informed consent. The study protocol was approved by the local ethics committees of the Medical University of Innsbruck, Austria (AN2015-0303 357/4.3), and of the Heart and Diabetes Center North-Rhine Westphalia, Ruhr University of Bochum, Germany (AZ 52/2016), the methods were carried out in accordance with the relevant guidelines and regulations, and the subjects gave written informed consent.

Pathway analysis. Information about analytes and the pathways in which they are involved was based on https://www.metaboanalyst.ca/ and on the Kyoto Encyclopedia of Genes and Genomes (KEGG).

\section{Data availability}

The datasets generated and analyzed during the current study are given as supplementary material (Supplemental Table 2).

Received: 31 July 2019; Accepted: 11 May 2020;

Published online: 02 June 2020

\section{References}

1. Anderson, P. A. et al. Contemporary outcomes after the Fontan procedure: a Pediatric Heart Network multicenter study. J Am Coll Cardiol 52(2), 85-98, https://doi.org/10.1016/j.jacc.2008.01.074 (2008).

2. Rychik, J. et al. Evaluation and management of the child and adult with Fontan circulation: a scientific statement from the American Heart Association. Circulation CIR0000000000000696, https://doi.org/10.1161/CIR.0000000000000696 (2019).

3. Giannakoulas, G. et al. Usefulness of natriuretic peptide levels to predict mortality in adults with congenital heart disease. Am J Cardiol 105(6), 869-73, https://doi.org/10.1016/j.amjcard.2009.11.041 (2010). 
4. Larsson, D. A., Meurling, C. J., Holmqvist, F., Waktare, J. E. \& Thilén, U. J. The diagnostic and prognostic value of brain natriuretic peptides in adults with a systemic morphologically right ventricle or Fontan-type circulation. Int J Cardiol 114(3), 345-51, https:// doi.org/10.1016/j.ijcard.2006.01.023 (2007).

5. Wang, C. H., Cheng, M. L. \& Liu, M. H. Amino acid-based metabolic panel provides robust prognostic value additive to B-natriuretic peptide and traditional risk factors in heart failure. Dis Markers 2018, 3784589, https://doi.org/10.1155/2018/3784589 (2018).

6. Siskos, A. P. et al. Interlaboratory reproducibility of a targeted metabolomics platform for analysis of human serum and plasma. Anal Chem 89(1), 656-665, https://doi.org/10.1021/acs.analchem.6b02930 (2017).

7. Ohuchi, H. et al. Abnormal glucose metabolism in patients with Fontan circulation: unique characteristics and associations with Fontan pathophysiology. Am Heart J 216, 125-35, https://doi.org/10.1016/j.ahj.2019.07.013 (2019).

8. Whiteside, W. et al. Altered cholesterol metabolism and hypocholesterolemia in patients with single ventricle following Fontan palliation. J Pediatr 171, 73-7, https://doi.org/10.1016/j.jpeds.2015.12.038 (2016).

9. Michel, M. et al. Targeted metabolomic analysis of serum phospholipid and acylcarnitine in the adult Fontan patient with a dominant left ventricle. Ther Adv Chronic Dis 11, 1-25, https://doi.org/10.1177/2040622320916031 (2020).

10. Schaffer, S. W., Shimada-Takaura, K., Jong, C. J., Ito, T. \& Takahashi, K. Impaired energy metabolism of the taurine-deficient heart. Amino Acids 48(2), 549-58, https://doi.org/10.1007/s00726-015-2110-2 (2016).

11. Kaplan, J. L. et al. Taurine deficiency and dilated cardiomyopathy in golden retrievers fed commercial diets. PLoS One 13(12), e0209112, https://doi.org/10.1371/journal.pone.0209112 (2018).

12. Ito, T., Schaffer, S. \& Azuma, J. The effect of taurine on chronic heart failure: actions of taurine against catecholamine and angiotensin II. Amino Acids 46(1), 111-19, https://doi.org/10.1007/s00726-013-1507-z (2014).

13. Müller-Hennessen et al. Metabolic profiles in heart failure due to non-ischemic cardiomyopathy at rest and under exercise. ESC Heart Fail 4(2), 178-89, https://doi.org/10.1002/ehf2.12133 (2017).

14. Neubauer, S. The failing heart - an engine out of fuel. N Engl J Med 356(11), 1140-51, https://doi.org/10.1056/NEJMra063052 (2007).

15. Murphy, J. M., Murch, S. J. \& Ball, R. O. Proline is synthesized from glutamate during intragastric infusion but not during intravenous infusion in neonatal piglets. J Nutr 126(4), 878-86, https://doi.org/10.1093/jn/126.4.878 (1996).

16. Wakabayashi, Y., Iwashima, A., Yamada, E. \& Yamada, R. Enzymological evidence for the indispensability of small intestine in the synthesis of arginine from glutamate. II. N-acetylglutamate synthase. Arch Biochem Biophys 291(1), 9-14, https://doi. org/10.1016/0003-9861(91)90098-4 (1991).

17. Nørrelund, H. et al. Abnormalities of whole body protein turnover, muscle metabolism and levels of metabolic hormones in patients with chronic heart failure. J Intern Med 260(1), 11-21, https://doi.org/10.1111/j.1365-2796.2006.01663.x (2006).

18. Qi, L. et al. Association between a genetic variant related to glutamic acid metabolism and coronary heart disease in individuals with type 2 diabetes. JAMA 310(8), 821-28, https://doi.org/10.1001/jama.2013.276305 (2013).

19. Nogueira Ade, C., Vale, R. G., Gomes, A. L. \& Dantas, E. H. The effect of muscle actions on the level of connective tissue damage. Res Sports Med 19(4), 259-70, https://doi.org/10.1080/15438627.2011.608046 (2011).

20. Koike, K. et al. Free 4-hydroxyproline content in serum of bedridden aged people is elevated due to fracture. Biol Pharm Bull 23(1), 101-3, https://doi.org/10.1248/bpb.23.101 (2000).

21. Visser, M. et al. The role of asymmetric dimethylarginine and arginine in the failing heart and its vasculature. Eur J Heart Fail 12(12), 1274-81, https://doi.org/10.1093/eurjhf/hfq158 (2010)

22. Massion, P. B., Feron, O., Dessy, C. \& Balligand, J. L. Nitric oxide and cardiac function: ten years after, and continuing. Circ Res 93(5), 388-98, https://doi.org/10.1161/01.RES.0000088351.58510.21 (2003).

23. Arimura, K. et al. Increased inactivation of nitric oxide is involved in coronary endothelial dysfunction in heart failure. Am J Physiol Heart Circ Physiol 280(1), H68-75, https://doi.org/10.1152/ajpheart.2001.280.1.H68 (2001).

24. Deanfield, J. E., Halcox, J. P. \& Rabelink, T. J. Endothelial function and dysfunction: testing and clinical relevance. Circulation 115(10), 1285-95, https://doi.org/10.1161/CIRCULATIONAHA.106.652859 (2007).

25. Andrews, K. L., Triggle, C. R. \& Ellis, A. N. O. and the vasculature: where does it come from and what does it do? Heart Fail Rev 7(4), 423-45, https://doi.org/10.1023/a:1020702215520 (2002).

26. Cooke, J. P. Does ADMA cause endothelial dysfunction? Arterioscler Thromb Vasc Biol 20(9), 2032-7, https://doi.org/10.1161/01. atv.20.9.2032 (2000).

27. Jud, P. et al. Homoarginine/ADMA ratio and homoarginine/SDMA ratio as independent predictors of cardiovascular mortality and cardiovascular events in lower extremity arterial disease. Sci Rep 8(1), 14197, https://doi.org/10.1038/s41598-018-32607-8 (2018).

28. Böger, R. H. et al. Supplementation of hypercholesterolaemic rabbits with L-arginine reduces the vascular release of superoxide anions and restores NO production. Atherosclerosis 117(2), 273-84, https://doi.org/10.1016/0021-9150(95)05582-h (1995).

29. Kielstein, J. T. et al. Asymmetric dimethylarginine, blood pressure, and renal perfusion in elderly subjects. Circulation 107(14), 1891-5, https://doi.org/10.1161/01.CIR.0000060496.23144.A7 (2003).

30. Miyazaki, H. et al. Endogenous nitric oxide synthase inhibitor: a novel marker of atherosclerosis. Circulation 99(9), 1141-6, https:// doi.org/10.1161/01.cir.99.9.1141 (1999).

31. Böger, R. H. et al. Biochemical evidence for impaired nitric oxide synthesis in patients with peripheral arterial occlusive disease. Circulation 95(8), 2068-74, https://doi.org/10.1161/01.cir.95.8.2068 (1997).

32. Surdacki, A. et al. Reduced urinary excretion of nitric oxide metabolites and increased plasma levels of asymmetric dimethylarginine in men with essential hypertension. J Cardiovasc Pharmacol 33(4), 652-8, https://doi.org/10.1097/00005344-199904000-00020 (1999).

33. Tutarel, O. et al. Asymmetrical dimethylarginine-more sensitive than NT-proBNP to diagnose heart failure in adults with congenital heart disease. PLoS One 7(3), e33795, https://doi.org/10.1371/journal.pone.0033795 (2012).

34. Martínez, Y. et al. The role of methionine on metabolism, oxidative stress, and diseases. Amino Acids 49(12), 2091-98, https://doi. org/10.1007/s00726-017-2494-2 (2017).

35. Picot, C. R. et al. Alterations in mitochondrial and cytosolic methionine sulfoxide reductase activity during cardiac ischemia and reperfusion. Exp Gerontol 41(7), 663-67, https://doi.org/10.1016/j.exger.2006.03.011 (2006).

36. Razavi, A. C. et al. Novel findings from a metabolomics study of left ventricular diastolic function: the Bogalusa Heart Study. J Am Heart Assoc 9(3), e015118, https://doi.org/10.1161/JAHA.119.015118 (2020).

37. Kolho, K. L., Pessia, A., Jaakkola, T., de Vos, W. M. \& Velagapudi, V. Faecal and serum metabolomics in paediatric inflammatory bowel disease. J Crohns Colitis 11(3), 321-34, https://doi.org/10.1093/ecco-jcc/jjw158 (2017).

38. Zyblewski, S. C. et al. Reduction in postoperative high-density lipoprotein cholesterol levels in children undergoing the Fontan operation. Pediatr Cardiol 33(7), 1154-9, https://doi.org/10.1007/s00246-012-0274-z (2012).

39. Whiteside, W., Tan, M., Yu, S. \& Rocchini, A. Low total, low-density lipoprotein, high-density lipoprotein, and non-high-density lipoprotein cholesterol levels in patients with complex congenital heart disease after Fontan palliation. J Pediatr 162(6), 1199-204, https://doi.org/10.1016/j.jpeds.2012.11.073 (2013).

40. Whiteside, W. et al. Altered cholesterol metabolism and hypocholesterolemia in patients with single ventricle following Fontan palliation. J Pediatr 171, 73-7, https://doi.org/10.1016/j.jpeds.2015.12.038 (2016).

41. Böger, R. H. et al. Asymmetric dimethylarginine (ADMA): a novel risk factor for endothelial dysfunction: its role in hypercholesterolemia. Circulation 98(18), 1842-7, https://doi.org/10.1161/01.cir.98.18.1842 (1998). 
42. von Haehling, S. et al. Elevated levels of asymmetric dimethylarginine in chronic heart failure: a pathophysiologic link between oxygen radical load and impaired vasodilator capacity and the therapeutic effect of allopurinol. Clin Pharmacol Ther 88(4), 506-12, https://doi.org/10.1038/clpt.2010.116 (2010).

43. Diller, G. P. et al. Exercise intolerance in adult congenital heart disease: comparative severity, correlates, and prognostic implication. Circulation 112(6), 828-35, https://doi.org/10.1161/CIRCULATIONAHA.104.529800 (2005).

44. Oechslin, E. et al. Systemic endothelial dysfunction in adults with cyanotic congenital heart disease. Circulation 112(8), 1106-12, https://doi.org/10.1161/CIRCULATIONAHA.105.534073 (2005).

45. Celermajer, D. S., Cullen, S. \& Deanfield, J. E. Impairment of endothelium-dependent pulmonary artery relaxation in children with congenital heart disease and abnormal pulmonary hemodynamics. Circulation 87(2), 440-6, https://doi.org/10.1161/01.cir.87.2.440 (1993).

46. Rhodes, J. et al. Effect of inhaled iloprost on the exercise function of fontan patients: A demonstration of concept. Int J Cardiol 168, 2435-2440, https://doi.org/10.1016/j.ijcard.2013.03.014 (2013).

47. Duncan, B. W. \& Desai, S. Pulmonary arteriovenous malformations after cavopulmonary anastomosis. Ann Thorac Surg 76(5), 1759-66, https://doi.org/10.1016/s0003-4975(03)00450-8 (2003).

48. Whitehead, K. K. et al. Status of systemic to pulmonary arterial collateral flow after the fontan procedure. Am J Cardiol 115(12), 1739-45, https://doi.org/10.1016/j.amjcard.2015.03.022 (2015).

49. Buga, G. M., Gold, M. E., Fukuto, J. M. \& Ignarro, L. J. Shear stress-induced release of nitric oxide from endothelial cells grown on beads. Hypertension 17(2), 187-93, https://doi.org/10.1161/01.hyp.17.2.187 (1991).

50. Aoki, M. et al. Endothelin-1 may play an important role in the Fontan circulation. Interact Cardiovasc Thorac Surg 26(3), 480-486, https://doi.org/10.1093/icvts/ivx378 (2018).

51. Ohuchi, H. et al. Positive pediatric exercise capacity trajectory predicts better adult Fontan physiology rationale for early establishment of exercise habits. Int J Cardiol 274, 80-87, https://doi.org/10.1016/j.ijcard.2018.06.067 (2018).

52. Dubowy, K. O., Baden, W., Bernitzki, S. \& Peters, B. A practical and transferable new protocol for treadmill testing of children and adults. Cardiol Young 18(6), 615-23, https://doi.org/10.1017/S1047951108003181 (2008).

\section{Acknowledgements}

The authors gratefully thank the laboratory team of the Erich and Hanna Klessmann Institut, Bad Oeynhausen, Germany, and the team of the "Kinderlabor" Innsbruck, Austria (Claudia Ertl, Ulrike Eichinger-Öttl, Christine Kluckner), for supporting our work by giving us the facilities to preanalyze and to store the serum samples; the team of the laboratory for exercise capacity testing in the Heart and Diabetes Center North Rhine-Westphalia, Bad Oeynhausen, Germany, for supporting us in exercise capacity testing and in blood drawing; and A.S. Knisely and E. Michel for expert comments on manuscript drafts.

\section{Author contributions}

M.M. designed the study, examined patients, prepared samples, analysed and interpreted results and wrote the manuscript; K.O.D. supervised lung function and exercise testing; K.O.D. and K.T.L. helped classify the patients; M.G.A. and U.M. performed biochemical assays and helped with statistical analysis of metabolomicsexamination results; S.S.B., K.T.L., and K.O.D. helped analyze amino acids and assisted in analysis of results; M.G.A., A.E., and U.M. provided the figures; all authors proofread the manuscript and read and approved the final version of the manuscript.

\section{Competing interests}

This work is part of the project "Metabolic aspects in Fontan patients", supported by a financial grant given to Dr. Michel from the "Tiroler Wissenschaftsförderung" (grant UNI-0404-2126). Dr. Michel has no further financial and no non-financial competing interests, or other interests that might be perceived to influence the results and/or discussion reported in this manuscript. All other authors declare no financial and no non-financial competing interests, or other interests that might be perceived to influence the results and/or discussion reported in this manuscript.

\section{Additional information}

Supplementary information is available for this paper at https://doi.org/10.1038/s41598-020-65852-x.

Correspondence and requests for materials should be addressed to M.M.

Reprints and permissions information is available at www.nature.com/reprints.

Publisher's note Springer Nature remains neutral with regard to jurisdictional claims in published maps and institutional affiliations.

(c) (i) Open Access This article is licensed under a Creative Commons Attribution 4.0 International License, which permits use, sharing, adaptation, distribution and reproduction in any medium or format, as long as you give appropriate credit to the original author(s) and the source, provide a link to the Creative Commons license, and indicate if changes were made. The images or other third party material in this article are included in the article's Creative Commons license, unless indicated otherwise in a credit line to the material. If material is not included in the article's Creative Commons license and your intended use is not permitted by statutory regulation or exceeds the permitted use, you will need to obtain permission directly from the copyright holder. To view a copy of this license, visit http://creativecommons.org/licenses/by/4.0/.

(C) The Author(s) 2020 